\title{
Generating vortex rings in Bose-Einstein condensates in the line-source approximation
}

\author{
M. Guilleumas, ${ }^{1}$ D. M. Jezek, ${ }^{2}$ R. Mayol,${ }^{1}$ M. Pi ${ }^{1}$ and M. Barranco ${ }^{1}$ \\ ${ }^{1}$ Departament d'Estructura i Constituents de la Matèria, Facultat de Física, Universitat de Barcelona, E-08028 Barcelona, Spain \\ ${ }^{2}$ Departamento de Física, Facultad de Ciencias Exactas y Naturales, Universidad de Buenos Aires \\ and Consejo Nacional de Investigaciones Cientificas y Técnicas, RA-1428 Buenos Aires, Argentina
}

(Received 5 December 2001; published 2 May 2002)

\begin{abstract}
We present a numerical method for generating vortex rings in Bose-Einstein condensates confined in axially symmetric traps. The vortex ring is generated using the line-source approximation for the vorticity, i.e., the curl of the superfluid velocity field is different from zero only on a circumference of a given radius located on a plane perpendicular to the symmetry axis and coaxial with it. The particle density is obtained by solving a modified Gross-Pitaevskii equation that incorporates the effect of the velocity field. We discuss the appearance of density profiles, the vortex core structure, and the vortex nucleation energy, i.e., the energy difference between vortical and ground-state configurations. This is used to present a qualitative description of the vortex dynamics.
\end{abstract}

DOI: 10.1103/PhysRevA.65.053609

\section{INTRODUCTION}

Since 1999, when vortex lines in a trapped Bose-Einstein condensate (BEC) were first experimentally obtained [1], their study has received great experimental and theoretical interest as it constitutes a clear signature of superfluidity effects in these confined systems. Some remarkable experimental achievements are, among others, the study of the dynamics of single vortex lines [2] and the formation of small [3] and large [4] vortex arrays. A review of the research done in this field is presented in Ref. [5]. It is worth noting the experimental situation in BEC regarding the formation and detection of vortices is at variance with that in He II droplets. For these drops, a paradigm of superfluid finite systems, the problem of nucleating vortices, and their stability has only been addressed very recently from the theoretical point of view (see Refs. [6-8]), and their experimental detection is still an open question.

Vortex rings are vortices whose core is a closed loop with quantized circulation around it [9]. They are complex topological structures that have attracted and will continue to attract some experimental and theoretical interest. Based on numerical simulations, different methods have been proposed to generate vortex rings in BEC. As in bulk liquid helium, vortex rings may be produced introducing an impurity in the condensate with a definite velocity, whose displacement causes a vortex ring [10]. Another mechanism [11] consists in using dynamical instabilities in the condensate to cause dark solitons to decay into vortex rings. A wellcontrolled method to produce vortex rings by electromagnetically induced atomic transitions in two-component condensates has also been put forward [12]. The method proposed in Ref. [11] has been successfully applied in Ref. [13] to generate vortex rings experimentally.

Rather than proposing a method that could be implemented to create a vortex ring experimentally, our aim here is to set up a numerical method simple yet accurate enough to generate quantized vortex rings with a definite radius $R$ in one-component condensates. We restrict our study to vortex states such that the divergence of their velocity is vanish-
PACS number(s): 03.75.Fi, 05.30.Jp, 32.80.Pj, 67.40.Vs

ingly small. This assumption yields analytical expressions for the velocity field, and provides a fair approximation to the superfluid flow around the vortex core for rings in the bulk of large condensates (Thomas-Fermi limit). We have considered large condensates at zero temperature and therefore, dissipation has not been taken into account. They are axially symmetric about the $z$ axis, and have the $z=0$ plane as the symmetry plane, and may host a vortex ring of radius $R$, coaxial with the symmetry axis of the trap, and placed on a plane at a distance $Z \geqslant 0$ from the symmetry plane. Although generalization to situations with more than one such vortex rings is straightforward, we have not considered this possibility.

This paper is organized as follows. In Sec. II, we present the method used to generate vortices. The way to obtain the velocity field of a vortex ring is described in Sec. III, and the explicit expressions of the velocity components are given in the Appendix. Section IV is devoted to the analysis of the particle density profiles and vortex nucleation energies, which allows a qualitative description of their dynamics. We also present results obtained from a completely different, more involved method we have set up to generate ring vortices that permits us to test the approximation of zero divergence of the velocity. Finally, a brief summary of the results is presented in Sec. V.

\section{ENERGY FUNCTIONAL}

We consider a weakly interacting Bose-condensed gas confined in a harmonic trap $V_{\text {ext }}(\mathbf{r})$ at zero temperature. In the Gross-Pitaevskii (GP) theory, the ground-state (GS) energy of the condensate is given by the functional [14]

$$
E[\Psi]=\int d \mathbf{r}\left[\frac{\hbar^{2}}{2 m}|\nabla \Psi|^{2}+V_{\text {ext }}(\mathbf{r})|\Psi|^{2}+\frac{g}{2}|\Psi|^{4}\right],
$$

where $\Psi(\mathbf{r})$ is the condensate wave function. The first term in Eq. (1) is the kinetic energy of the condensate, the second term is the harmonic oscillator energy arising from the trapping potential, and the third term is the mean-field interac- 
tion energy. The coupling constant is $g=4 \pi \hbar^{2} a / m$, where $a$ is the $s$-wave scattering length and $m$ is the atomic mass. The number of atoms in the condensate is $\int d \mathbf{r}|\Psi|^{2}=N$. The GS wave function is determined by solving the GP equation obtained minimizing the energy functional.

The wave function $\Psi$ can be written in terms of the particle density $\rho(\mathbf{r})=|\Psi(\mathbf{r})|^{2}$ and phase $S(\mathbf{r})$ as

$$
\Psi(\mathbf{r})=\sqrt{\rho(\mathbf{r})} \exp [i S(\mathbf{r})],
$$

and the superfluid velocity is given by $\mathbf{v}=(\hbar / m) \nabla S$. In this work we will use the equivalent quantum hydrodynamic description of the condensate in terms of the density and the superfluid velocity [14], since it allows a straightforward generalization of the energy functional Eq. (1) to include vortex states. Using Eq. (2) it follows that

$$
E[\Psi]=E_{0}[\rho]+E_{\text {kin }}^{\mathbf{v}}[\rho, \mathbf{v}] .
$$

The first term is only density dependent

$$
E_{0}[\rho]=\int d \mathbf{r}\left[\frac{\hbar}{2 m}|\nabla \sqrt{\rho}|^{2}+V_{\mathrm{ext}}(\mathbf{r}) \rho+\frac{g}{2} \rho^{2}\right] .
$$

The first term in $E_{0}[\rho]$ is the quantum kinetic energy. The second term of the energy functional Eq. (3) corresponds to the kinetic energy associated with the flow velocity $\mathbf{v}$, and is given by

$$
E_{\mathrm{kin}}^{\mathbf{v}}[\rho, \mathbf{v}]=\frac{1}{2} m \int d \mathbf{r} \rho(\mathbf{r}) \mathbf{v}^{2}(\mathbf{r}) .
$$

The GS wave function in the absence of vortices has a spatially constant phase and therefore zero velocity. It can be obtained by minimizing $E_{0}[\rho]$. We consider condensates with positive scattering length and axially symmetric traps $V_{\text {ext }}(\mathbf{r})=m\left[\omega_{\perp}^{2}\left(x^{2}+y^{2}\right)+\omega_{z}^{2} z^{2}\right] / 2\left[=m\left(\omega_{\perp}^{2} r^{2}+\omega_{z}^{2} z^{2}\right) / 2\right.$ in cylindrical $(r, z)$ coordinates], with different values of the asymmetry parameter $\lambda=\omega_{z} / \omega_{\perp}$. The trap harmonic frequency $\omega_{\perp}$ provides a length scale for the system, $a_{\perp}$ $=\left(\hbar / m \omega_{\perp}\right)^{1 / 2}$. We will use $a_{\perp}, \hbar \omega_{\perp}$, and $N / a_{\perp}^{3}$ as units of length, energy, and density, respectively.

If we consider large condensates in which the ThomasFermi (TF) approximation holds [14], the quantum kinetic energy can be neglected compared to the interaction energy in Eq. (4), and the GS density of the condensate in the absence of vortices is given by $\rho_{0}(r, z)=\mu\left(1-r^{2} / R_{\mathrm{TF}}^{2}\right.$ $\left.-z^{2} / Z_{\mathrm{TF}}^{2}\right) / g$ in the region where this expression is positive, and zero elsewhere. The TF extents of the condensate in the radial and axial directions are $R_{\mathrm{TF}}=\left(2 \mu / m \omega_{\perp}^{2}\right)^{1 / 2}$ and $Z_{\mathrm{TF}}$ $=R_{\mathrm{TF}} / \lambda$. The chemical potential $\mu$ is fixed by normalization $\mu=\hbar \omega_{\perp}\left(15 \lambda a N / a_{\perp}\right)^{2 / 5} / 2$. We recall that the validity of the Thomas-Fermi approximation is guaranteed if $N a / a_{\perp} \gg 1$.

We turn now our attention to the case of condensates with vortex states characterized by a given irrotational velocity field associated with a nonvanishing quantized circulation. The total energy of the system is given by the energy functional Eq. (3). If the velocity field is known, for a given number of particles the density profile can be obtained minimizing Eq. (3). This yields the equation

$$
\left(-\frac{\hbar^{2} \boldsymbol{\nabla}^{2}}{2 m}+V_{\mathrm{ext}}(\mathbf{r})+g|\psi(\mathbf{r})|^{2}+\frac{1}{2} m \mathbf{v}^{2}\right) \psi(\mathbf{r})=\mu \psi(\mathbf{r}),
$$

where $\psi=\sqrt{\rho}$ is the modulus of the complex wave function Eq. (2). This is the GP equation expressed in terms of the hydrodynamic variables.

In the presence of a quantized vortex, the density of the system drops to zero at its core, whose size is characterized by a healing length $\xi$. For very large condensates, the healing length can be approximated by $\xi=\left(8 \pi \rho_{0} a\right)^{-1 / 2}$, where $\rho_{0}$ is the density of the condensate before creating the vortex. For a centered vortex line, in the TF approximation $\rho_{0}=\mu / g$ and the corresponding healing length $\xi_{0}$ is

$$
\frac{\xi_{0}}{R_{\mathrm{TF}}}=\left(\frac{a_{\perp}}{R_{\mathrm{TF}}}\right)^{2},
$$

with $\xi_{0} \ll a_{\perp} \ll R_{T F}$. In this approximation a local healing length can be defined [15] as $\xi(r, z)$ $=\xi_{0} / \sqrt{1-\left(r / R_{\mathrm{TF}}\right)^{2}-\left(z / Z_{\mathrm{TF}}\right)^{2}}$. Note that the size of the core is larger for a vortex in the low-density region.

\section{SUPERFLUID VELOCITY FIELD}

The velocity field around a straight vortex line has an analytical expression when the vortex is along the symmetry axis $[5,16]$. Approximate analytical expressions can be found in the case of vortex lines off the symmetry axis in large and very elongated, quasi-two dimensional condensates (see Ref. [17] and references therein). However, vortex lines generally bend in three-dimensional condensates $[5,18]$ which renders impracticable an analytical treatment of the velocity field.

In the case of quantized vortex rings we proceed as Schwarz and Jang have done in the case of He II [19] (see also Ref. [20]). For a vortex ring characterized by the values of $(R, Z)$ already defined, circulation number $n=1,2, \ldots$, and quantum circulation $k_{0}=n \mathrm{~h} / \mathrm{m}$, we write the vorticity in the line-source approximation:

$$
\boldsymbol{\omega}=k_{0} \delta(r-R) \delta(z-Z) \hat{\boldsymbol{\phi}}
$$

where $(r, z, \phi)$ are the cylindrical coordinates, and $\hat{\boldsymbol{\phi}}$ is the unit vector in the azimuthal direction $\hat{\boldsymbol{\phi}}=(-\sin \phi, \cos \phi, 0)$. The superfluid velocity field that arises from this distributed vorticity fulfills $\boldsymbol{\omega}=\boldsymbol{\nabla} \times \mathbf{v}$. Hence, the velocity field around the vortex is irrotational except on the vorticity line, where the density of the condensate is zero. If $\boldsymbol{\nabla} \cdot \mathbf{v}=0$ to a good approximation [21], a velocity vector potential $\mathbf{A}(\mathbf{r})$ can be introduced such that $\mathbf{v}=\boldsymbol{\nabla} \times \mathbf{A}$. If the vorticity is specified, $\mathbf{A}(\mathbf{r})$ is determined by the equation

$$
\boldsymbol{\omega}=\boldsymbol{\nabla} \times(\boldsymbol{\nabla} \times \mathbf{A}),
$$

whose integral solution is [19] $\mathbf{A}(\mathbf{r})=A_{0}(r, z) \hat{\boldsymbol{\phi}}$, with 


$$
A_{0}(r, z)=\frac{k_{0}}{4 \pi} R \int_{0}^{2 \pi} \frac{\cos \phi^{\prime} d \phi^{\prime}}{\sqrt{r^{2}+R^{2}-2 r R \cos \phi^{\prime}+(z-Z)^{2}}} .
$$

The radial and $z$ components of the velocity are obtained as

$$
\begin{gathered}
v_{r}=-\frac{\partial A_{0}}{\partial z}, \\
v_{z}=\frac{1}{r} \frac{\partial}{\partial r}\left(r A_{0}\right),
\end{gathered}
$$

whereas the azimuthal component of the velocity field around the vortex ring Eq. (8) is zero. We give in the Appendix the general expressions of $A_{0}, v_{r}$, and $v_{z}$ written in terms of hypergeometric functions [22].

For a confined system, the existence of a boundary and the fact that the density is inhomogeneous may have an effect on the actual velocity field [23]. In our case, since the condensate extends up to "infinite distances," there is no need to introduce any image vortex to ensure that there is no particle flow across the boundary due to the superfluid motion. Moreover, it has been shown [15] that in the TF limit the corrections to the velocity due to density inhomogeneities can be safely neglected. Thus, we approximate the velocity field by Eqs. (10) and (11) (see the Appendix), but have restricted ourselves to study vortex ring configurations $(R, Z)$ whose vorticity line is inside the domain where the density is positive in the TF approximation, i.e., only vortex rings $(R, Z)$ satisfying the condition $\left(R / R_{\mathrm{TF}}\right)^{2}+\left(Z / Z_{\mathrm{TF}}\right)^{2}$ $<1$ are considered. We call the $(R, Z)$ line defined by the condition $\left(R / R_{\mathrm{TF}}\right)^{2}+\left(Z / Z_{\mathrm{TF}}\right)^{2}=1$ the TF boundary. Once the superfluid velocity field has been fixed, the density profile of the condensate is obtained solving Eq. (6).

\section{RESULTS}

We present numerical results for large condensates in the Thomas-Fermi limit hosting a quantized vortex ring with circulation number equal to one (nucleating vortex rings with $n>1$ is energetically less favorable [9,24]). For a singly quantized vortex ring configuration $(R, Z)$ with vorticity given by Eq. (8) and circulation number $n=1$, we have computed the velocity field, Eq. (11), and have obtained the density profile of the confined condensate by solving Eq. (6) in cilyndrical coordinates using the imaginary time method [25]. Note that although we are in the TF limit, we do solve the complete GP equation to obtain the density profiles.

Figure 1 shows density profiles in the $z=0$ plane as a function of $r$ for the experimental parameters of Ref. [13], that is, $N=3 \times 10^{5}$ atoms of ${ }^{87} \mathrm{Rb}$ (scattering length $a$ $\left.=5.82 \times 10^{-9} \mathrm{~cm}\right)$ in a spherical trap with $\omega_{\perp} / 2 \pi$ $=7.8 \mathrm{~Hz}$. We have plotted two configurations with a vortex ring located in $Z=0$ having a radius $R=2 a_{\perp}$ (dotted line), and $R=4 a_{\perp}$ (dotted-dashed line), respectively. The density profile of the condensate without a vortex is also shown (solid line).

The density is zero on the vorticity line, as seen in the

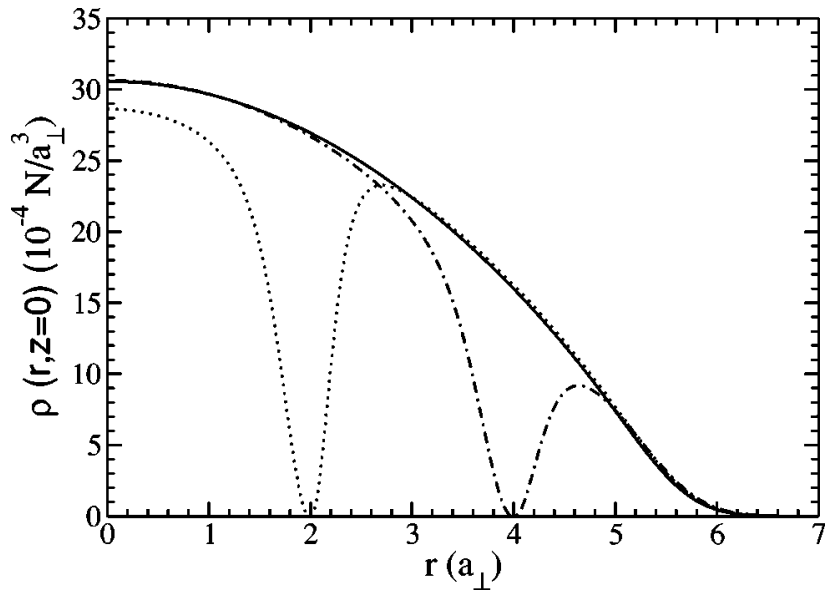

FIG. 1. Density profile as a function of $r$ at $z=0$ for a condensate in a spherical trap with the experimental parameters of Ref. [13] $\left(a N / a_{\perp}=440\right)$ hosting a singly quantized vortex ring at $Z$ $=0$. The dotted line corresponds to a vortex ring with $R=2 a_{\perp}$, and the dot-dashed line to a vortex ring with $R=4 a_{\perp}$. The solid line is the GS density profile in the absence of vortices. The densities have been normalized to one.

density profiles. As expected, the size of the core is of the order of the healing length. Indeed, for this condensate one has $R_{\mathrm{TF}} \simeq 5.8 a_{\perp}$ and $\xi_{0} \sim 0.17 a_{\perp}$. Assuming that the vortex diameter is twice the local healing length, and using the TF expressions we get as core diameters the values $2 \xi(2,0)$ $=0.37 a_{\perp}$, and $2 \xi(4,0)=0.47 a_{\perp}$. One can see from Fig. 1 that the core sizes are in agreement with these estimates. When the radius of the vortex ring increases, the core lies at a lower density region and therefore its size increases.

To study the vortex ring energetics we have chosen a larger condensate made of $N=10^{6}$ atoms of ${ }^{87} \mathrm{Rb}$ confined in an axially symmetric trap with axial frequency $\omega_{z} / 2 \pi$ $=220 \mathrm{~Hz}$ and three different geometries, namely, spherical, disk shaped, and cigar shaped, with $\lambda=1, \sqrt{8}$, and 0.2 , respectively. For this condensate, we show in Fig. 2 several equidensity lines (arbitrary values) in the $y=0$ plane. The top panel corresponds to the cigar-shaped trap $\left(\lambda=0.2, R_{\mathrm{TF}}\right.$ $\left.=8.7 a_{\perp}, Z_{\mathrm{TF}}=43.3 a_{\perp}\right)$, the middle panel to the spherically symmetric trap $\left(\lambda=1, R_{\mathrm{TF}}=Z_{\mathrm{TF}}=10.2 a_{\perp}\right)$, and the bottom panel to the disk-shaped trap $\left(\lambda=\sqrt{8}, R_{\mathrm{TF}}=45.5 a_{\perp}, Z_{\mathrm{TF}}\right.$ $\left.=11.3 a_{\perp}\right)$. The $x$ and $z$ axes are in units of $a_{\perp}$. For all geometries, the vortex ring configuration is $\left(R=3.1 a_{\perp}, Z\right.$ $=0)$. The intersection between the vorticity line and the $y$ $=0$ plane appears as two dark dots, indicating the steep density depression around the vortex core. The presence of the vorticity causes a drastic distortion of the density with respect to the GS profile. Yet, for large condensates it is a rather local effect, as can be seen from Fig. 2, and also from Fig. 1 for the smaller condensate.

The nucleation of a vortex has an energy cost, since the energy of a condensate with a vortex is always larger than the energy of the condensate without it, $E_{\mathrm{GS}}$. The vortex nucleation energy, $E-E_{\mathrm{GS}}$, corresponding to the condensate of the spherical trap in Fig. 2 is plotted in Fig. 3 as a function of $Z$ for different values of $R$. At fixed $R$, the nucleation energy is maximum when the ring is in the $z=0$ plane, and it 


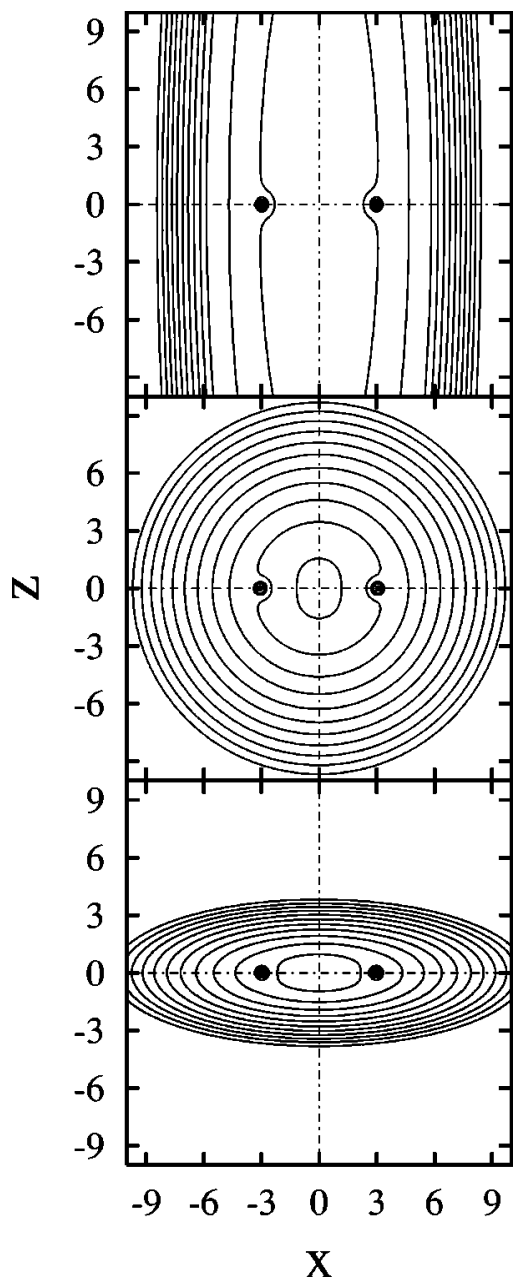

FIG. 2. Vortex ring state equidensity lines, in arbitrary units, plotted in the $y=0$ plane (axes are in units of $a_{\perp}$ ). The ring is at $\left(R=3.1 a_{\perp}, Z=0\right)$ in a condensate with $N=10^{6}$ atoms of ${ }^{87} \mathrm{Rb}$, confined in an axially symmetric trap with axial frequency $\omega_{z} / 2 \pi$ $=220 \mathrm{~Hz}$ and different trap geometries. The top panel corresponds to a cigar-shaped trap $\left(\lambda=0.2, R_{\mathrm{TF}}=8.7 a_{\perp}, Z_{\mathrm{TF}}=43.3 a_{\perp}\right)$, the middle panel to a spherically symmetric trap $\left(\lambda=1, R_{\mathrm{TF}}\right.$ $\left.=10.2 a_{\perp}\right)$, and the bottom panel to a disk-shaped trap $(\lambda$ $=\sqrt{8}, R_{\mathrm{TF}}=45.5 a_{\perp}, Z_{\mathrm{TF}}=11.3 a_{\perp}$ ).

decreases as $Z$ increases, since displacing the ring outwards implies that the superfluid flow affects less atoms in the condensate. Note that to have the vorticity line within the TF boundary, the $R$ curves end at different values of $Z / Z_{\mathrm{TF}}$ given by $\sqrt{1-\left(R / R_{\mathrm{TF}}\right)^{2}}$. The nucleation energy does not vanish when the ring is located on the TF boundary as there still exists a superfluid velocity field which produces some effect. Since we treat the superfluid velocity as an external field, the nucleation energy of very superficial vortex configurations may be somewhat overestimated. This drawback is apparent beyond the TF boundary, and shows up as a long-tailed nucleation energy as a function of either $Z / Z_{T F}$ or $R / R_{T F}$, although it goes eventually to zero.

The nucleation energy is plotted in Fig. 4 as a function of $R$ for different $Z$ values. The smaller ring we have considered has $R / R_{\mathrm{TF}} \sim 0.1$. The middle panel corresponds to the same spherical condensate as in Fig. 3. Besides, the top panel cor-

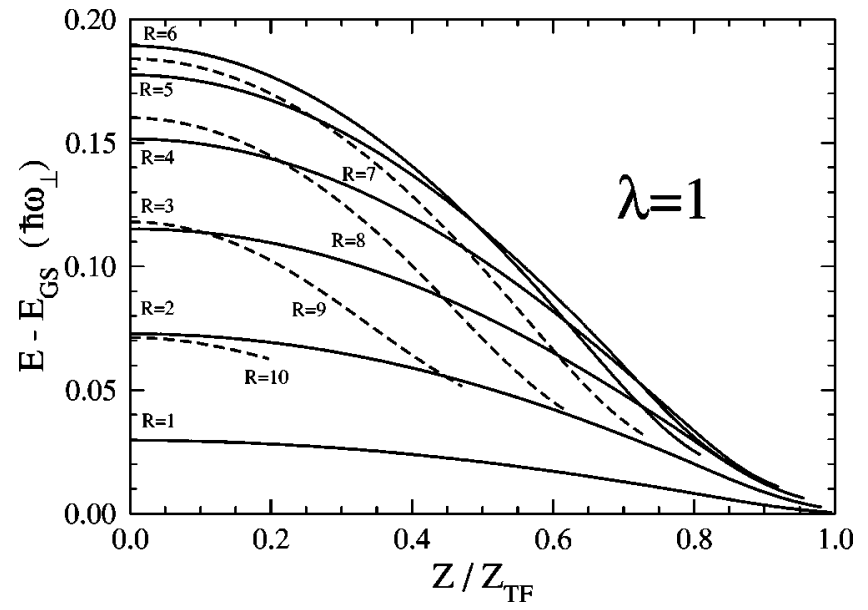

FIG. 3. Nucleation energy of a vortex ring $(R, Z)$ as a function of $Z$ for different values of the radius. The labels of the curves are in units of $a_{\perp}$. The condensate has $N=10^{6}$ atoms of ${ }^{87} \mathrm{Rb}$ and it is confined in a spherically symmetric trap with frequency $\omega_{z} / 2 \pi$ $=220 \mathrm{~Hz}$. The TF radius is $R_{\mathrm{TF}}=Z_{\mathrm{TF}}=10.2 a_{\perp}$.

responds to the cigar-shaped trap, and the bottom panel to the disk-shaped trap. The top curve in all panels corresponds to vortex rings with $Z=0$, and for a given trap geometry (fixed panel), the higher the curve, the lower the $Z$. As in Fig. 3 , we have only described vortex ring configurations inside the TF boundary. For this reason, for a given $Z$ value the corresponding nucleation energy curve stops at the value $R / R_{\mathrm{TF}}=\sqrt{1-\left(Z / Z_{\mathrm{TF}}\right)^{2}}$.

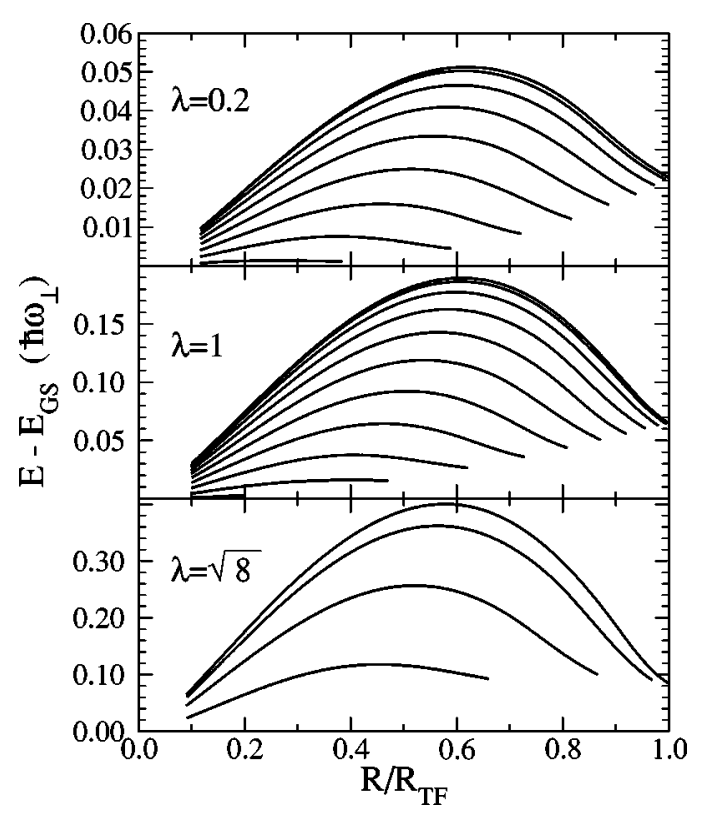

FIG. 4. Nucleation energy of a vortex $\operatorname{ring}(R, Z)$ as a function of $R$ for different values of $Z$. The condensate and trap geometries are those of Fig. 2. The top panel corresponds to the cigar-shaped trap, the middle panel to the spherically symmetric trap, and the bottom panel to the disk-shaped trap. From top to bottom, and in units of $a_{\perp}$, the curves correspond to $Z=0$ to 40 in $Z$ steps of 5 (top panel); $Z=0$ to 10 in $Z$ steps of 1 (middle panel); $Z=0,1,2$, and 3 (bottom panel). 
Figure 4 shows that for each asymmetry parameter $\lambda$, given a $Z$ there exists a vortex configuration corresponding to a certain radius $R$ that maximizes the nucleation energy. This radius increases as $Z$ decreases. In the $(R, Z)$ twodimensional configuration space, the nucleation energy $E(R, Z)-E_{\mathrm{GS}}$ has only one maximum at $\left(R_{\mathrm{eq}} / R_{\mathrm{TF}} \simeq 0.6, Z\right.$ $=0)$. It is in agreement with the results obtained in Ref. [26] for a spherically symmetric trap using a simplified model of a vortex ring.

This maximum corresponds to the only stationary, although unstable, vortex ring configuration (equivalent to a vortex line nucleated along the $z$ axis), whose location is almost independent of $\lambda$ but its value depends on it, decreasing as $\lambda$ does. This means that the energy to nucleate a vortex ring characterized by the values of $(R, Z)$ is lower the more elongated is the trap.

For each asymmetry parameter, the "iso-nucleation energy" lines in the $(R, Z)$ configuration space corresponding to a value not too far from the maximum value are closed curves around the maximum, and the allowed ring radii are in the range defined by the intersection of a straight line representing the given nucleation energy and the $Z=0$ curve in Fig. 4. If we relax the condition that the vorticity line must be inside the TF boundary, this should be always like this [26]. Otherwise, the isoenergy lines eventually do not close. Yet, the allowed ring radii are determined in a similar way.

We have considered the vortex ring as if it were a static object. However, in a trapped condensate a vortex ring will move with a velocity that results from the interplay between the effect caused by the inhomogeneity of the condensate and self-induced effects arising from its own local curvature $[5,9,26]$. A qualitative analysis of the dynamics of coaxial vortex rings can be carried out from Figs. 3 and 4, as proposed in Ref. [26], and tested in a real-time dynamics for two-component condensates $[12,26]$. It can be seen that a coaxial vortex ring oscillates inside the condensate, moving towards the surface and instead of being annihilated at the boundary, the ring generates a background flow in the rest of the cloud that draws it back towards the center. The ring dynamics in a trapped condensate results in an oscillation along the symmetry axis of the trap. The radius of the vortex ring grows or shrinks depending on its $Z$ position, in such a way that the ring motion almost follows a trajectory with constant energy in the $(R, Z)$ configuration space.

Finally, we have used a completely different method, numerically more involved, to generate quantized vortex rings. It does not make any assumption on the superfluid velocity field, nor on the radius or position of the vortex ring either, and can be used to test the results we have obtained based on the validity of the approximation $\boldsymbol{\nabla} \cdot \mathbf{v}=0$.

We have proceeded by analogy with the case of a vortex line, and have added a constraint to the energy functional that forces to form a toroidallike hole in the condensate with a flux of atoms around its core with zero azimuthal velocity component. We have used as constraining operator the angular momentum about the $\hat{\boldsymbol{\phi}}$ axis, i.e., $\hat{\boldsymbol{\phi}} \cdot \mathbf{L} \equiv L_{\boldsymbol{\phi}}=i \hbar\left(r \partial_{z}\right.$ $\left.-z \partial_{r}\right)[27]$.

Introducing the functional

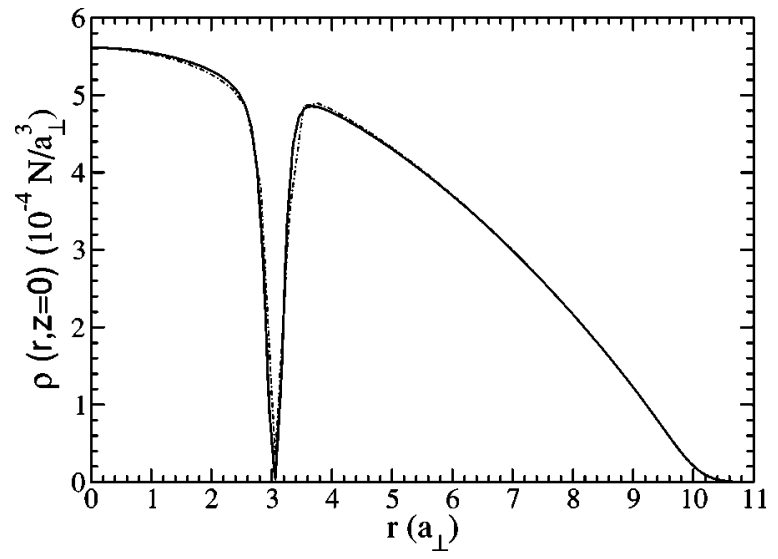

FIG. 5. Density profile as function of $r$ at $z=0$ for the $N=10^{6}$ condensate in a spherically symmetric trap with a singly quantized vortex ring. The solid line corresponds to the density profile that minimizes the functional Eq. (12) with constraint $\Omega_{\phi}=0.2 \omega_{\perp}$. The dashed line is the density obtained imposing a ring vorticity at $(R$ $=3.1 a_{\perp}, Z=0$ ). The densities have been normalized to one.

$$
\begin{aligned}
E[\Psi]= & \int d \mathbf{r}\left[\frac{\hbar^{2}}{2 m}|\nabla \Psi|^{2}+V_{\text {ext }}(\mathbf{r})|\Psi|^{2}+\frac{g}{2}|\Psi|^{4}\right] \\
& +\Omega_{\phi} \int d \mathbf{r} \Psi^{*} L_{\phi} \Psi,
\end{aligned}
$$

where the Lagrange multiplier $\Omega_{\phi}$ can be understood as the local angular velocity about the azimuthal direction, we have obtained the associated GP equation. For a given $\Omega_{\phi}$, we have solved the partial differential equations obeyed by the real and imaginary part of the condensate wave function, which are coupled by the presence of the constraint. These equations have been discretized on a $(r, z)$ mesh using seven-point formulas to represent the differential operators, and have been solved using again the imaginary time method [25].

Taking as an example $\Omega_{\phi}=0.2 \omega_{\perp}$, we have obtained a coaxial vortex ring configuration with its vorticity located on the $z=0$ plane whose equidensity lines cannot be distinguished from these represented in the middle panel of Fig. 2 at the scale of this figure (whose parameters were chosen before to allow us to make this statement).

Figure 5 presents a comparison between the two methods. It shows the particle density profile as a function of $r$ at $z$ $=0$ for the spherical condensate of Fig. 3. The solid line is the configuration that minimizes Eq. (12). The density goes to zero in $R=3.1 a_{\perp}$, and the vortex ring has an energy equal to $37.164 \hbar \omega_{\perp}$. The dashed line is the configuration obtained by the method of Sec. II with the ring vorticity Eq. (8) placed in $\left(R=3.1 a_{\perp}, Z=0\right)$ which has an energy of $37.167 \hbar \omega_{\perp}$. Thus, the energy and density profile of both configurations are almost identical, and the velocity fields are also in good agreement.

We have also checked that minimizing the functional Eq. (12) yields quantized ring vortices with $n=1$. To this end, we have proceeded to a direct numerical integration of the circulation $\oint \mathbf{v} \cdot d \mathbf{l}$ using several arbitrary closed paths in the 


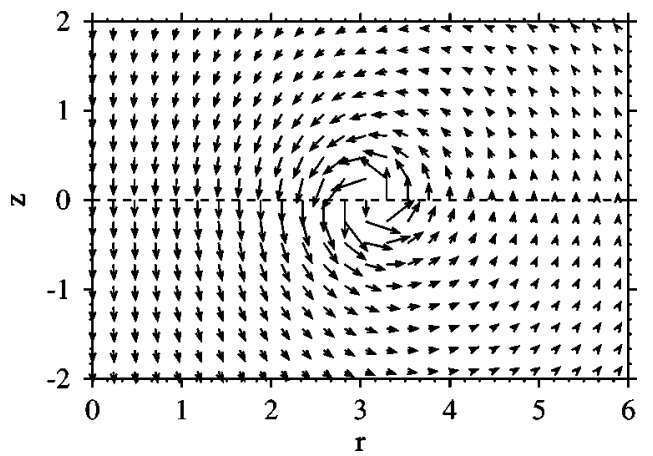

FIG. 6. Current field around the vortex core corresponding to the vortex ring state calculated taking $\Omega_{\phi}=0.2 \omega_{\perp}$ in Eq. (12). The current is in arbitrary units and the axes are in units of $a_{\perp}$.

$(r, z)$ "plane." We have found that the circulation for paths enclosing the vortex core is $h / m$ with a good accuracy, and is zero otherwise. The velocity field has been calculated from the wave function recalling that the current field is $\mathbf{j}(\mathbf{r})$ $=\rho \mathbf{v}=(\hbar / 2 m i)\left[\Psi^{*} \nabla \Psi-\left(\boldsymbol{\nabla} \Psi^{*}\right) \Psi\right]$. The current field $\mathbf{j}(\mathbf{r})$ in the $y=0$ plane is plotted in arbitrary units in Fig. 6. The axes are in units of $a_{\perp}$. It can be clearly distinguished in this figure the position of the vortex ring core at $Z=0$ and $R$ $\simeq 3.1 a_{\perp}$.

\section{SUMMARY}

We have developed a method to study vortex rings hosted in confined condensates in the TF limit. It provides analytical expressions for the superfluid velocity field that satisfy the irrotational condition and the quantization of the circulation of the superfluid flow. The velocity around the vortex core is introduced as an external field in the Gross-Pitaevskii energy functional, which is minimized to obtain the wave function of the condensate.

Using this method, we have computed the density profile and nucleation energy of vortex rings in one-component condensates. We have found that the size of the vortex ring core is of the order of the healing length as in the case of vortex lines. The presence of the vortex causes a sizeable distortion of the density, as atoms are pushed off the core, but the effect is rather local.

The analysis of the dependence of the nucleation energy on the position and radius of the ring allows one to conclude that a Hamiltonian dynamics will lead to oscillations of the vortex ring along the symmetry axis of the condensate, increasing and decreasing its radius in accordance with previous predictions. A dissipative dynamics would cause the vortex to decay, for example disappearing across the border of the condensate, as occurs for vortex lines [28].

The method provides a handleable way to generate vortex rings in confined condensates that can be used as a starting point to study the dynamics. A detailed calculation of the vortex ring dynamics in a confined condensate, either Hamiltonian or dissipative, is beyond the scope of the present paper and will be developed elsewhere.

\section{ACKNOWLEDGMENTS}

This work has been performed under Grant Nos. PB981247 from DGESIC, Spain, and 2000SGR-00024 from Generalitat de Catalunya. D.M.J. acknowledges the CONICET (Argentina) and the Generalitat de Catalunya ACI program for financial support.

\section{APPENDIX}

In this appendix we give the expressions and method we have used to obtain the velocity field. From Eqs. (10)-(11) one has

$$
\begin{aligned}
v_{r}(r, z)= & \frac{k_{0} R(z-Z)}{\pi\left[(r+R)^{2}+(z-Z)^{2}\right]^{3 / 2}} \\
& \times \int_{0}^{\pi / 2} \frac{2 \cos ^{2} \phi-1}{\left(1-s \cos ^{2} \phi\right)^{3 / 2}} d \phi,
\end{aligned}
$$

where we have defined $s \equiv 4 r R /\left[(r+R)^{2}+(z-Z)^{2}\right]$. The integral Eq. (A1) is written in terms of hypergeometric functions [22] yielding

$$
\begin{aligned}
v_{r}(r, z)= & \frac{k_{0} R(z-Z)}{2\left[(r+R)^{2}+(z-Z)^{2}\right]^{3 / 2}} \\
& \times[F(3 / 2,3 / 2 ; 2 ; s)-F(1 / 2,3 / 2 ; 1 ; s)] .
\end{aligned}
$$

Similarly,

$$
\begin{aligned}
v_{z}(r, z)= & \frac{k_{0} R}{2 r\left[(r+R)^{2}+(z-Z)^{2}\right]^{1 / 2}} \\
& \times[F(3 / 2,1 / 2 ; 2 ; s)-F(1 / 2,1 / 2 ; 1 ; s)] \\
& +\frac{k_{0} R}{2\left[(r+R)^{2}+(z-Z)^{2}\right]^{3 / 2}} \\
& \times[(r+R) F(1 / 2,3 / 2 ; 1 ; s) \\
& \left.-(r+2 R) F(3 / 2,3 / 2 ; 2 ; s)+\frac{3 R}{2} F(5 / 2,3 / 2 ; 3 ; s)\right]
\end{aligned}
$$

and

$$
\begin{aligned}
A_{0}(r, z)= & \frac{k_{0} R}{2\left[(r+R)^{2}+(z-Z)^{2}\right]^{1 / 2}} \\
& \times[F(3 / 2,1 / 2 ; 2 ; s)-F(1 / 2,1 / 2 ; 1 ; s)] .
\end{aligned}
$$

The hypergeometric functions entering Eqs. (A2)-(A4) can be written in terms of the complete elliptic integrals $\mathbf{E}$ and $\mathbf{K}$ [22] as follows: 


$$
\begin{gathered}
F(1 / 2,1 / 2 ; 1 ; s)=\frac{2}{\pi} \mathbf{K}(\sqrt{s}), \\
F(1 / 2,3 / 2 ; 1 ; s)=F(3 / 2,1 / 2 ; 1 ; s)=\frac{2}{\pi} \frac{\mathbf{E}(\sqrt{s})}{1-s}, \\
F(3 / 2,1 / 2 ; 2 ; s)=F(1 / 2,3 / 2 ; 2 ; s)=\frac{4}{\pi s}[\mathbf{K}(\sqrt{s})-\mathbf{E}(\sqrt{s})],
\end{gathered}
$$

$$
\begin{gathered}
F(3 / 2,3 / 2 ; 2 ; s)=\frac{4}{\pi s}\left[\frac{\mathbf{E}(\sqrt{s})}{1-s}-\mathbf{K}(\sqrt{s})\right] \\
F(5 / 2,3 / 2 ; 3 ; s)=\frac{16}{3 \pi s^{2}}\left[\frac{2-s}{1-s} \mathbf{E}(\sqrt{s})-2 \mathbf{K}(\sqrt{s})\right] .
\end{gathered}
$$

To evaluate $\mathbf{E}$ and $\mathbf{K}$ we have used polynomial approximations [29].
[1] M.R. Matthews, B.P. Anderson, P.C. Haljan, D.S. Hall, C.E. Wieman, and E.A. Cornell, Phys. Rev. Lett. 83, 2498 (1999).

[2] B.P. Anderson, P.C. Haljan, C.E. Wieman, and E.A. Cornell, Phys. Rev. Lett. 85, 2857 (2000).

[3] K.W. Madison, F. Chevy, W. Wohlleben, and J. Dalibard, Phys. Rev. Lett. 84, 806 (2000).

[4] J.R. Abo-Shaeer, C. Raman, J.M. Vogels, and W. Ketterle, Science 292, 476 (2001).

[5] A.L. Fetter and A.A. Svidzinsky, J. Phys.: Condens. Matter 13, R135 (2001).

[6] G.H. Bauer, R.J. Donnelly, and W.F. Vinen, J. Low Temp. Phys. 98, 47 (1995).

[7] F. Dalfovo, R. Mayol, M. Pi, and M. Barranco, Phys. Rev. Lett. 85, 1028 (2000).

[8] R. Mayol, M. Pi, M. Barranco, and F. Dalfovo, Phys. Rev. Lett. 87, 145301 (2001).

[9] R. J. Donnelly, Quantized Vortices in Helium II (Cambridge University Press, Cambridge, England, 1991).

[10] B. Jackson, J.F. McCann, and C.S. Adams, Phys. Rev. A 60, 4882 (1999).

[11] D.L. Feder, M.S. Pindzola, L.A. Collins, B.I. Schneider, and C.W. Clark, Phys. Rev. A 62, 053606 (2000).

[12] J. Ruostekoski and J.R. Anglin, Phys. Rev. Lett. 86, 3934 (2001).

[13] B.P. Anderson, P.C. Haljan, C.A. Regal, D.L. Feder, L.A. Collins, C.W. Clark, and E.A. Cornell, Phys. Rev. Lett. 86, 2926 (2001).
[14] F. Dalfovo, S. Giorgini, L. Pitaevskii, and S. Stringari, Rev. Mod. Phys. 71, 463 (1999).

[15] E. Lundh and P. Ao, Phys. Rev. A 61, 063612 (2000).

[16] F. Dalfovo and S. Stringari, Phys. Rev. A 53, 2477 (1996).

[17] M. Guilleumas and R. Graham, Phys. Rev. A 64, 033607 (2001).

[18] J.J. García-Ripoll and V.M. Pérez-García, Phys. Rev. A 63, 041603(R) (2001).

[19] K.K. Schwarz and P.S. Jang, Phys. Rev. A 8, 3199 (1973).

[20] D.M. Jezek, M. Pi, M. Barranco, R.J. Lombard, and M. Guilleumas, J. Low Temp. Phys. 112, 303 (1998).

[21] Note that since the density is not uniform, the assumption $\boldsymbol{\nabla} \cdot \mathbf{v} \approx 0$ does not imply that the system is incompressible.

[22] I. S. Gradshteyn and I. M. Ryzhik, Table of Integrals, Series and Products (Academic Press, New York, 1980).

[23] J.R. Anglin, e-print cond-mat/0110389; Phys. Rev. A (to be published).

[24] T. Winiecki, J.F. McCann, and C.S. Adams, Europhys. Lett. 48, 476 (1999).

[25] M. Pi, A. Emperador, M. Barranco, and F. Garcias, Phys. Rev. B 63, 115316 (2001).

[26] B. Jackson, J.F. McCann, and C.S. Adams, Phys. Rev. A 61, 013604 (1999).

[27] Actually, we have used the Hermitian form $L_{\phi}=(\hat{\boldsymbol{\phi}} \cdot \mathbf{L}$ $+\mathbf{L} \cdot \hat{\boldsymbol{\phi}}) / 2$.

[28] P.O. Fedichev and G.V. Shlyapnikov, Phys. Rev. A 60, R1779 (1999).

[29] M. Abramowitz and I. Stegun, Handbook of Mathematical Functions (Dover, New York, 1970). 